\title{
THE FIRST ALLWISE PROPER MOTION DISCOVERY: WISEA J070720.50+170532.7
}

\author{
Edward L. Wright ${ }^{1}$, J. Davy Kirkpatrick ${ }^{2}$, Christopher R. Gelino ${ }^{2}$, Sergio Fajardo-Acosta ${ }^{2}$, Gregory Mace $^{1}$, \\ Peter R. Eisenhardt ${ }^{3}$, Daniel Stern ${ }^{3}$, Ian S. McLean ${ }^{1}$, M. F. Skrutskie ${ }^{4}$, Apurva Oza ${ }^{4}$, M. J. Nelson ${ }^{4}$, \\ Michael C. Cushing ${ }^{5}$, I. Neill Reid ${ }^{6}$, Michele Fumagalli ${ }^{7}, 8,10$, and Adam J. Burgasser ${ }^{9}$ \\ ${ }^{1}$ UCLA Astronomy, P.O. Box 951547, Los Angeles, CA 90095-1547, USA; wright@ astro.ucla.edu \\ 2 Infrared Processing and Analysis Center, California Institute of Technology, Pasadena, CA 91125, USA \\ ${ }^{3}$ Jet Propulsion Laboratory, California Institute of Technology, 4800 Oak Grove Drive, Pasadena, CA 91109, USA \\ ${ }^{4}$ Department of Astronomy, University of Virginia, Charlottesville, VA 22904, USA \\ ${ }^{5}$ Department of Physics and Astronomy, University of Toledo, 2801 West Bancroft Street, Toledo, OH 43606-3328, USA \\ ${ }^{6}$ STScI, Baltimore, MD 21218, USA \\ ${ }^{7}$ Carnegie Observatories, 813 Santa Barbara Street, Pasadena, CA 91101, USA \\ ${ }^{8}$ Department of Astrophysics, Princeton University, Princeton, NJ 08544-1001, USA \\ ${ }^{9}$ University of California San Diego, 9500 Gilman Drive, La Jolla, CA 92093, USA \\ Received 2013 October 23; accepted 2013 December 6; published 2014 February 10
}

\begin{abstract}
While quality checking a new motion-aware co-addition of all 12.5 months of Wide-field Infrared Survey Explorer (WISE) data, we found that the source WISE J070720.48+170533.0 moved 0'9 9 in six months. Backtracking this motion allowed us to identify this source as 2MASS J07071961+1705464, with several entries in the USNO B

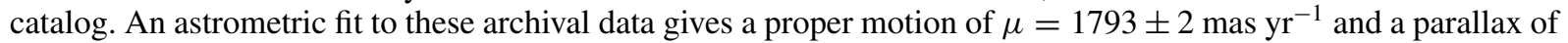
$\varpi=35 \pm 42$ mas. Photometry from WISE, 2MASS, and the POSS can be fit reasonably well by a blackbody with $T=3658 \mathrm{~K}$ and an angular radius of $4.36 \times 10^{-11}$ radians. No clear evidence of $\mathrm{H}_{2}$ collision-induced absorption is seen in the near-infrared. An optical spectrum shows broad deep CaH bands at 638 and $690 \mathrm{~nm}$, broad deep Na D at $598.2 \mathrm{~nm}$, and weak or absent TiO, indicating that this source is an ultra-subdwarf $\mathrm{M}$ star with a radial velocity $v_{\text {rad }} \approx-21 \pm 18 \mathrm{~km} \mathrm{~s}^{-1}$ relative to the Sun. Given its apparent magnitude, the distance is about $39 \pm 9 \mathrm{pc}$ and the tangential velocity is probably $\approx 330 \mathrm{~km} \mathrm{~s}^{-1}$, but a more precise parallax is needed to be certain.
\end{abstract}

Key words: brown dwarfs - infrared: stars - solar neighborhood - stars: individual (WISEA J070720.50+ 170532.7) - stars: late-type - stars: low-mass

Online-only material: color figures, supplemental data

\section{INTRODUCTION}

The Wide-field Infrared Survey Explorer (WISE; Wright et al. 2010) mapped the entire sky between 2010 January 14 and 2010 July 17 in four thermal infrared bands centered at 3.4, 4.6, 12, and $22 \mu \mathrm{m}$, then continued on to map the entire sky again prior to 2011 February 1. On 2010 August 7, the outer cryogen tank ran out of solid hydrogen coolant, and the three-band cryo phase began, where the $22 \mu \mathrm{m}$ channel was lost and the $12 \mu \mathrm{m}$ channel had much reduced sensitivity due to the background radiated by the optics, which warmed to $45 \mathrm{~K}$. On 2010 September 30, the inner cryogen tank ran out of solid hydrogen, and the two-band post-cryo phase began. This phase lasted until 2011 February 1, with only the 3.4 and $4.6 \mu \mathrm{m}$ channels operating. This last phase of the mission, which lasted for four months, was never co-added or cataloged. Only single frame detections were found to search for near-Earth objects as part of the NEOWISE project (Mainzer et al. 2011).

The AllWISE project is an effort to co-add all the WISE frames, find peaks in these co-adds, and then fit for the properties of the source or sources responsible for each peak by doing profile fit photometry using the pixels on each frame that cover the position of the peak. While doing this fit, the AllWISE project is allowing for motion in addition to the usual parameters of the position and the fluxes in each of the four bands.

Previous efforts to find nearby stars with the WISE data have concentrated on color-selected searches. Kirkpatrick et al. (2011) found over one hundred brown dwarfs using the color

\footnotetext{
${ }^{10}$ Hubble Fellow.
}

cut $W 1-W 2>2$, and many more have been studied in other papers such as Mace et al. (2013). These color-selected searches have found many high proper motion objects. However, several papers have searched for moving objects using the Two Micron All Sky Survey (2MASS) match ( $3^{\prime \prime}$ radius) in the WISE catalog as a veto (Gizis et al. 2011; Liu et al. 2011; Thompson et al. 2013). With AllWISE, we seek to find previously missed high motion objects using positive associations between observations over the full timespan of WISE. This has been done by Luhman (2013) for objects bright enough to appear in WISE single frame detections, leading to the discovery of a brown dwarf binary only $2 \mathrm{pc}$ from the Sun. AllWISE is doing a similar search using co-added frames to reach the full sensitivity of WISE. Kirkpatrick et al. (2014) will discuss a large sample of AllWISE motion discoveries, but this brief note describes one of the most interesting AllWISE motion selected sources whose colors are similar to hundreds of millions of WISE objects.

\section{OBSERVATIONS}

\subsection{Astrometry and Photometry}

\subsubsection{AllWISE}

The AllWISE catalog is formed by fitting the pixels in the individual frames with a model containing eight parameters, which are the position, motion, and fluxes for a point source: $\left(\alpha, \delta, \mu_{\alpha}=\cos \delta d \alpha / d t, \mu_{\delta}, F_{W 1}, F_{W 2}, F_{W 3}\right.$, and $\left.F_{W_{4}}\right)$. This model is convolved with the point-spread function of WISE, compared to the pixel data, and then adjusted to get the best fit. The position and motion parameters, which have a non-linear 
effect on the pixel values, are often hard to fit in a robust and stable way, so the fitting procedure first finds the best fit with $\mu_{\alpha}=\mu_{\delta}=0$, which just repeats the procedure used for the previous WISE catalogs, and then uses this as a starting point for an iterative adjustment to find the best fitting motion. In addition, the first $\mu=0$ solution is used to generate the source designation and when extracting fluxes from individual frames that go into the multi-epoch photometry (MEP) database (see Section 2.2).

The AllWISE catalog entry for W0707+1705 has the designation WISEA J070720.50+170532.7. The first position solution, for $\mu_{\alpha}=\mu_{\delta}=0$, gives $\alpha=106.8354461 \pm 0$ '.0446, $\delta=17.0924311 \pm 0.0431$. The magnitudes obtained in the fixed position solution are $W 1234=12.646(24), 12.502(25)$, $>11.701$, and $>8.719$. The contamination and confusion flags are all zero, indicating that the detection is not affected by known artifacts. This position is then used to initialize the simultaneous solution for the position at epoch MJD $=55400$, the proper motion, and the four WISE magnitudes, giving $\alpha=106.8354766 \pm 0.0459, \delta=17.0924049 \pm 0^{\prime \prime} .0446$,

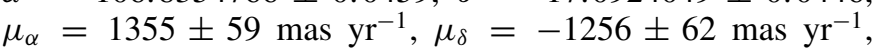
and $W 1234=12.660(23), 12.512(25), 12.430(530)$, and $>$ 8.702. Note that the $W 3$ flux is essentially identical in both solutions but one is slightly more than $2 \sigma$ while the other is slightly less and thus quoted as an upper limit. The quoted motion assumes that the parallax is zero, and a star with a non-zero parallax will have a motion that differs from the true proper motion by as much as $\pm 4 \varpi$ since the parallactic movement in six months is $\pm 2 \varpi$ and the time interval is one half year.

Note that the error on the motion of W0707+1705 is considerably smaller than the error on the position. This happens because the reference frame error terms cancel out in the position difference. For the optimal range of magnitudes, $8<W 1<10$, with high signal-to-noise ratio, but not saturated, the motion errors can be as small as $35 \mathrm{mas}_{\mathrm{yr}}^{-1}, 1 \sigma, 1$ axis. Thus, the

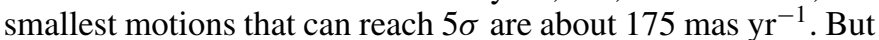
for fainter sources, the noise terms dominate the motion error, so for $W 1 \approx 15$ the errors are $10 \times$ larger, giving a $5 \sigma$ threshold of about 1750 mas $\mathrm{yr}^{-1}$. The upper limit on motions that can be detected by AllWISE is still being investigated and will be discussed further in Kirkpatrick et al. (2014). But Barnard's star and Kapteyn's star are recovered as moving sources, even though they are quite saturated.

\subsubsection{Separated WISE Epochs}

In order to combine the WISE astrometry with archival data from earlier surveys, we use the position from the WISE All-sky catalog, $\alpha=106.8353609 \pm 00^{\prime \prime} .0898, \delta=17.0925197 \pm 0^{\prime \prime} .0889$ at a mean MJD of 55287.577 .

Since W0707+1705 is bright enough to be easily detected on individual frames, the mid-average of the positions found on 12 post-cryogenic detections is used, giving $\alpha=106.835568 \pm$ $0^{\prime} .09, \delta=17.092344 \pm 0.09$ at a mean MJD of 55479.133.

\subsubsection{Cross-identifications}

The AllWISE motion, backtracked ten years to the 2MASS epoch, predicts a position northwest of the WISE position. Indeed, an easily seen source in the 2MASS (Skrutskie et al. 2006) image northwest of the WISE position is not seen in the WISE image, while the WISE source is not seen in the 2MASS image. This source is 2MASS J07071961+1705464 with $\alpha=$ $106.831722 \pm 00^{\prime} .07, \delta=17.096224 \pm 0 . ' 07$, and magnitudes
Table 1

Astrometric Data [J2000]

\begin{tabular}{lcrcrc}
\hline \hline MJD & $\begin{array}{c}\alpha \\
\left.{ }^{\circ}\right)\end{array}$ & $\begin{array}{c}\sigma_{\alpha} \\
(\mathrm{mas})\end{array}$ & $\begin{array}{c}\delta \\
\left({ }^{\circ}\right)\end{array}$ & $\begin{array}{c}\sigma_{\delta} \\
(\mathrm{mas})\end{array}$ & Source \\
\hline 33957.456 & 106.814542 & 195 & 17.113712 & 79 & USNO B1.0 \\
48986.792 & 106.829188 & 333 & 17.098708 & 333 & POSS2 B FITS WCS \\
49277.000 & 106.829498 & 114 & 17.098514 & 25 & USNO B1.0 \\
50405.411 & 106.830604 & 333 & 17.097333 & 333 & POSS2 R FITS WCS \\
50923.000 & 106.831139 & 999 & 17.096873 & 999 & USNO B1.0 \\
51496.920 & 106.831671 & 333 & 17.096272 & 333 & POSS2 IR FITS WCS \\
51579.657 & 106.831722 & 70 & 17.096224 & 70 & 2MASS \\
51902.000 & 106.832112 & 122 & 17.095854 & 122 & UCAC4 \\
52064.000 & 106.832222 & 88 & 17.095716 & 63 & CMC14 \\
55287.577 & 106.835361 & 90 & 17.092520 & 90 & WISE all-sky \\
55479.133 & 106.835568 & 90 & 17.092344 & 90 & WISE postcryo \\
56545.350 & 106.836599 & 50 & 17.091278 & 50 & FanCam \\
\hline
\end{tabular}

$J H K_{s}=13.473(25), 13.045(29)$, and 12.923(17) on MJD = 51579.657.

W0707+1705 appears in the Carlsberg Meridian Catalogue 14 (Evans et al. 2002, CMC14). This observation also gives an AB magnitude of $15.811 \pm 0.17$ in the SDSS $r$ band, where the quoted error is the external uncertainty for sources with $r_{\mathrm{AB}} \approx 16$ (Evans et al. 2002). The UCAC4 (Zacharias et al. 2013) contains W0707+1705 as entry 536-039034. No proper motion is given. The $579-642 \mathrm{~nm}$ magnitude is $15.881 \pm 0.30$ on the Vega system. These CCD-based surveys give better photometry than photographic plates, but W0707+1705 is close to the survey limit in both cases.

The USNO B1.0 catalog (Monet et al. 2003) contains several entries that appear to refer to W0707+1705. Entries 10710154281, 1070-0147856, and 1070-0147865 have positions and epochs that are consistent with the movement of W0707+1705, while the entry 1070-0147855 appears to be W0707+1705 in the second-epoch blue plate associated with an incorrect identification on the first-epoch blue plate. In addition to the astrometric data, we take photometric values of $B=$ $17.66,17.70$, and 18.33; $R=15.46$; and $I=14.97$ from these entries. Some of these entries have large position errors (999 max) but all are reasonably consistent with the astrometric solution.

Magnitudes from WISE, 2MASS, and the USNO-B are on the Vega system.

In addition to the WISE, 2MASS, and USNO B astrometry, data were also obtained by fitting parabolae to the data around the peak of the source on FITS postage stamp images from the Digitized Sky Survey maintained at the Space Telescope Science Institute. These values rely on the World Coordinate System in the FITS header and the pixel positions reported by the SAOImage DS9 FITS viewer (Joye \& Mandel 2003). For these values, the error is taken to be one-third of a pixel in the FITS image, typically 333 mas.

\subsubsection{Fan Mountain Observatory/FanCam}

$Y J H K_{s}$ photometry and astrometry of W0707+1705 were obtained on 2013 September 5 with FanCam, a HAWAII-1 based near-infrared imager operating at the University of Virginia's Fan Mountain 31 inch telescope (Kanneganti et al. 2009). Results are given in Tables 1 and 2.

All of the astrometric data used in this paper are listed in Table 1. Figure 1 shows images of W0707+1705 from 1952 to 2010. 



Figure 1. $2 \times 2$ arcmin images of the field near WISE $0707+1705$. North is up and east is to the left. Arrows point to the high proper motion star at each epoch.

Table 2

Photometric Observations of W0707+1705

\begin{tabular}{lcc}
\hline \hline Filter & Magnitude & Instrument \\
\hline$B$ & $17.9 \pm 0.25$ & USNO B \\
$579-642 \mathrm{~nm}$ & $15.881 \pm 0.3$ & UCAC4 \\
$r_{\mathrm{AB}}$ & $15.811 \pm 0.17$ & CMC14 \\
$R$ & $15.46 \pm 0.33$ & USNO B \\
$I$ & $14.97 \pm 0.33$ & USNO B \\
$Y(\mathrm{MKO})$ & $13.90 \pm 0.01$ & FanCam \\
$J$ & $13.47 \pm 0.01$ & FanCam \\
$J$ & $13.473 \pm 0.025$ & 2MASS \\
$H$ & $13.08 \pm 0.01$ & FanCam \\
$H$ & $13.045 \pm 0.029$ & 2MASS \\
$K_{s}$ & $12.91 \pm 0.01$ & FanCam \\
$K_{s}$ & $12.923 \pm 0.017$ & 2MASS \\
$W 1$ & $12.660 \pm 0.023$ & WISEA \\
$W 2$ & $12.512 \pm 0.025$ & WISEA \\
$W 3$ & $12.430 \pm 0.530$ & WISEA \\
$W 4$ & $>8.702$ & WISEA \\
\hline
\end{tabular}

Notes. All magnitudes except $r_{\mathrm{AB}}$ are Vega magnitudes and the $J H K_{s}$ bands use 2MASS filters. The $Y$-band calibration uses the Hamuy et al. (2006) transformation of $Y-K_{s}$ versus $J-K_{s}$.

\subsubsection{Ultraviolet}

W0707+1705 was not detected by Galaxy Evolution Explorer (Martin et al. 1999), but its position was observed during the All-sky Imaging Survey. Examining the expected position of W0707+1705 on the near-UV FITS image of the field shows it is at least 17 times fainter than the nearby source at $\alpha=106.837313, \delta=17.099230$, which has a near-UV $\mathrm{AB}$ magnitude of 18.32 , so W0707+1705 is fainter than 21.4 or $<10^{-5} \mathrm{Jy}$ at $0.24 \mu \mathrm{m}$.

\subsubsection{Astrometric Fit}

A parallax and proper motion fit to the data in Table 1 gives $\mu_{\alpha}=1228 \pm 3 \operatorname{mas~yr}^{-1}, \mu_{\delta}=-1307 \pm 1 \operatorname{mas~yr}^{-1}$, and a parallax of $\varpi=35 \pm 42$ mas. The residuals give a $\chi^{2}$ per degree of freedom $=8.72 / 19$, so the errors are reasonable if perhaps conservative. This best fit solution to a long time baseline is consistent with the six month baseline AllWISE catalog motion within $2 \sigma: \Delta \mu_{\alpha}=-127 \pm 59$ and $\Delta \mu_{\delta}=-51 \pm 62 \mathrm{mas} \mathrm{yr}^{-1}$. Figure 2 shows the residuals after a position and proper motion fit is removed.

\subsubsection{Photometric Fit}

The magnitudes from WISE, 2MASS, and the USNO B were fit to a blackbody using a least sum of absolute value of the error criterion. Monet et al. (2003) state that the photometric calibration of the USNO B catalog is of marginal quality due to the lack of an all-sky network of faint photometric calibration stars, and that the standard deviation of the fit to the existing calibration network is 0.25 mag. Thus, we used assumed uncertainties of 0.33 mag for the USNO B except in the blue, where there are three consistent estimates so a 0.25 mag uncertainty was used. For WISE and 2MASS the actual magnitude uncertainties were replaced by $0.1 \mathrm{mag}$ to allow for the crudeness of the blackbody model. The error for the $\mathrm{CMC} 14$ was rounded up to $0.2 \mathrm{mag}$ for the same reason. The photometric points and the best fit model with $T=3658 \mathrm{~K}$ and a solid angle of $10^{-20.224} \mathrm{sr}$ are shown in Figure 3. This solid angle corresponds to the Sun at a distance of $517 \mathrm{pc}$ with an angular radius of $9 \mu$ as.

A more pertinent comparison is the moderately metal-poor Barnard's star (GJ 699), which has a measured radius of $0.1867 \pm 0.0012 R_{\odot}$ (Boyajian et al. 2012). This would put 


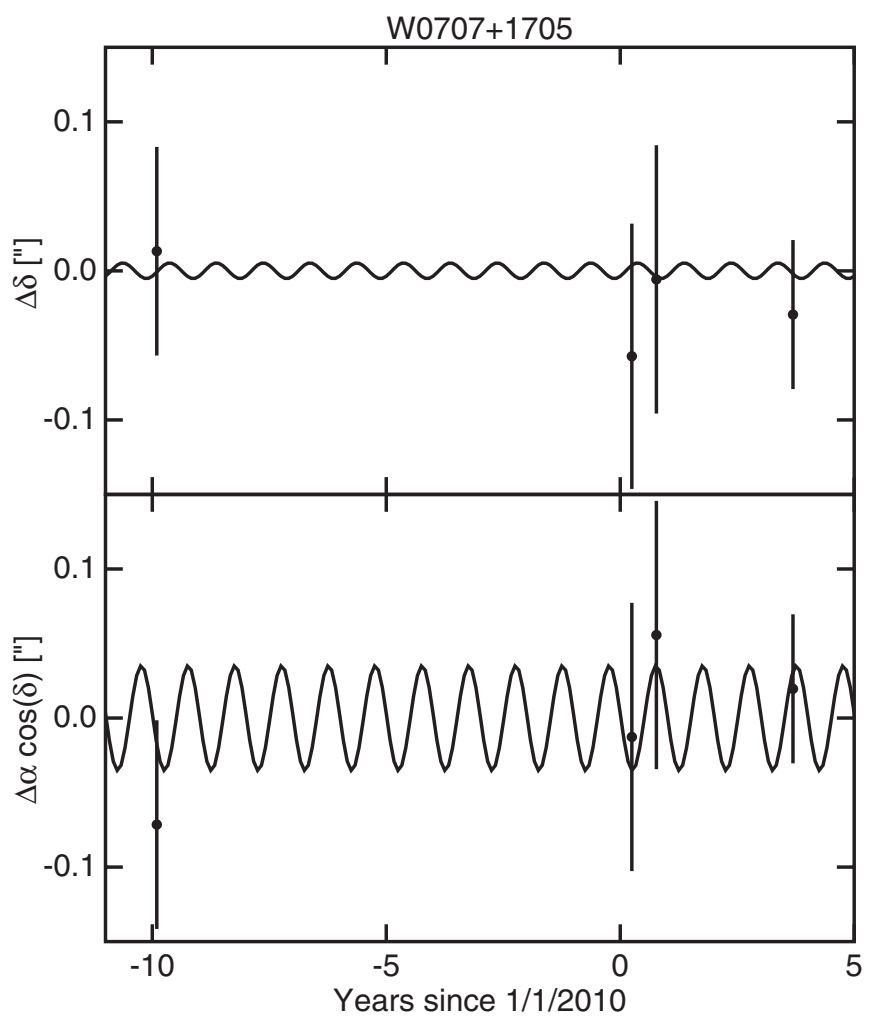

Figure 2. Astrometric residuals after a proper-motion-only fit is removed. Only the more precise data from 2MASS, WISE, and FanCam have been plotted to reduce clutter.

W0707+1705 at a distance of $97 \mathrm{pc}$. A blackbody fit to the fluxes of Barnard's star, similar to the one we did for W0707+1705, gives a radius of 587 mas. The distance of Barnard's star is $1.8 \mathrm{pc}$, so if W0707+1705 were like Barnard's star its distance would be $117 \mathrm{pc}$.

The near-infrared fluxes are clearly not suppressed by $\mathrm{H}_{2}$ collision-induced absorption, as would be expected for a cool white dwarf with a hydrogen atmosphere like LHS 3250 (Harris et al. 1999). In fact, the slight excess over the fit at $J$ and $H$ might indicate the presence of $\mathrm{H}^{-}$absorption.

\subsection{Variability}

A comparison of the 2MASS and FanCAM magnitudes shows that W0707+1705 has had a constant brightness over 13 years. But a new product in the AllWISE data release allows one to check for variability over hourly or six month intervals. In addition to checking for the motion of sources when generating the catalog, AllWISE is also producing a very large database of MEP. This MEP database contains the flux estimates in each WISE band for each frame that covers a source. These individual frame flux estimates have been generated in past releases and used to set the variability flag, but not stored. However, for AllWISE the single frame values are kept in a searchable database available at irsa.ipac.caltech.edu. These flux estimates are from the forced photometry at the position of the source, assuming it is celestially fixed, not the moving position from the proper motion fit. Since the AllWISE catalog and reject table together contain more than $10^{9}$ sources, and each source is observed on an average of 36 frames, the MEP database has $42,759,337,365$ rows and thus should not be used for large area blind surveys for variable objects. But it is easy to collect the individual frame data for a source or a list of sources.

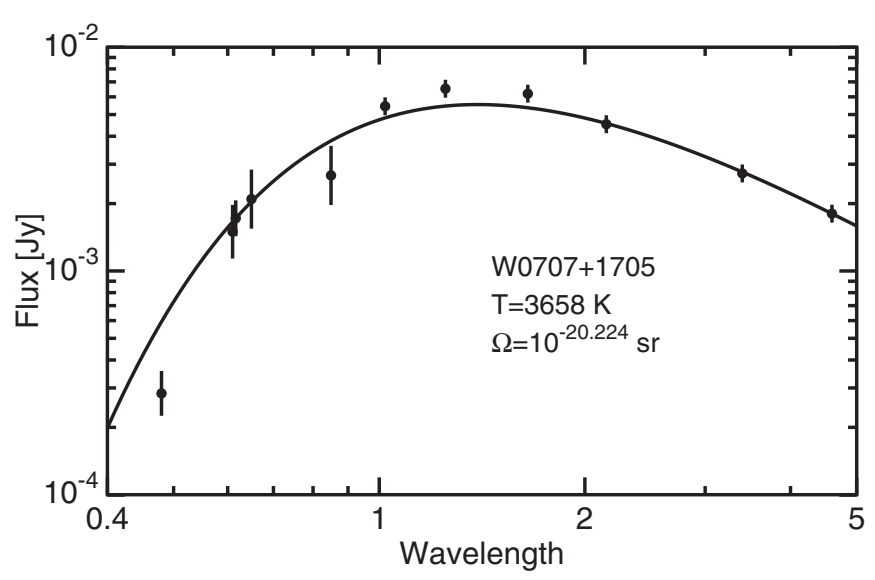

Figure 3. Photometric points and a blackbody fit.

The MEP database differs from the previously released single frame detection list in one important regard: the single frame detections had to be $>5 \sigma$ in at least one band in a single frame. For sources that are just over $5 \sigma$ in $W 1$ in the co-add of 30 frames, which includes a large fraction of cataloged WISE sources, the individual frames will usually only yield upper limits. The single frame detection list will only have the few frames with upward noise fluctuations, leading to a biased view of the source flux. Because most fluxes in the MEP will be insignificant, it is a good idea to use the flux and flux sigma columns for analysis. The fluxes are quoted in data numbers, which are close to the actual quantization in the downloaded images, but the data numbers have been scaled to correct for the different pixel and frame sensitivities, so $1 \mathrm{dn}$ always corresponds to a consistent flux. The zero-point magnitudes that correspond to a flux of $1 \mathrm{dn}$ are $W 1234=20.5,19.5,18$, and 13, the same as the zero points for the all-sky release given in Table 1 of Section 2.3.f of the Explanatory Supplement.

Figure 4 shows the flux values and uncertainties in the four WISE bands for the 25 frames covering W0707+1705. Fitting a constant flux to each band gives $1383.10 \pm 8.38$ dn with a $\chi^{2}$ of 54.8 for 24 degrees of freedom in $W 1$, $627.93 \pm 5.72$ dn with $\chi^{2} /$ dof $=49.9 / 24$ in $W 2,162.7 \pm 67.3$ dn with $\chi^{2} /$ dof $=7.0 / 12$ in $W 3$, and $11.24 \pm 17.29 \mathrm{dn}$ with $\chi^{2} /$ dof $=14.2 / 12$ in $W 4$. These uncertainties are statistical only and do not include systematic terms such as the uncertainty in the zero point. The $W 3$ value corresponds to $W 3=12.47$ with a signal-to-noise ratio of 2.42:1, providing no evidence for a warm dust disk around W0707+1705.

Moderate improvements in the $\chi^{2}$ for $W 1$ and $W 2$ can be made by fitting the epochs separately. $\Delta \chi^{2}=6.4$ in $W 1$ with the later epoch, $\Delta m, 0.033$ mag fainter. In $W 2, \Delta \chi^{2}$ is 14.9 with the second epoch 0.076 mag fainter. Given all the changes in the detector operating conditions as the telescope warmed up, these results are only weak evidence for a small amount of long term variability over a six month time span. But the comparison of 2MASS and FanCam over a 13 year span indicates that W0707+1705 is probably not varying.

\subsection{Spectroscopy}

We obtained an optical spectrum on UT 2013 September 3 with the Double Spectrograph at Palomar Mountain Observatory through partial cloud coverage during morning twilight. This spectrum is plotted in Figure 5. No calibration star could be observed so the sensitivity calibration is based on calibrations 




Figure 4. Plot of multi-epoch photometry data for W0707+1705. In each band, the left and right panels cover the first and second epochs of observation, separated by six months. Fluxes are in data numbers. $1 \mathrm{dn}$ corresponds to magnitudes $W 1234=20.5,19.5,18$, and 13 .

over the previous month, and atmospheric features have not been divided out. The $3680 \mathrm{~K}$ blackbody fit to the photometric data is a reasonable match to the trend of the spectrum, but the flux scaling is arbitrary.

The sodium D doublet of W0707+1705 is deep and broad with an equivalent width of $\approx 1.2 \mathrm{~nm}$ and an FWHM for the merged lines of $\approx 1.4 \mathrm{~nm}$. It shows a radial velocity $<100 \mathrm{~km} \mathrm{~s}^{-1}$, as seen in Figure 6. The Ca II infrared triplet indicates $v_{\text {rad }}=-45 \pm$ $18 / \mathrm{km} \mathrm{s}^{-1}$ with respect to the Earth, or $-21 \mathrm{~km} \mathrm{~s}^{-1}$ with respect to the Sun. Figure 7 gives an expanded view of the Ca II infrared triplet.

The $\mathrm{TiO}$ band at $715 \mathrm{~nm}$ is much weaker than the $690 \mathrm{~nm}$ $\mathrm{CaH}$ band, and the telluric water band at $718.5 \mathrm{~nm}$ may provide all the structure seen in this region of the spectrum. Weak TiO to $\mathrm{CaH}$ is an indicator of an extreme (esdM) or ultra-subdwarf (usdM), according to Lépine et al. (2007). Figure 8 compares the spectrum of W0707+1705 to the spectral standards in Lépine et al. (2007). In this region, W0707+1705 resembles a usdM2 or usdM3.5 star. The usdM3.5 spectral standard is LHS 325, while the usdM2 standard is LHS 1691. Their resemblance to W0707+1705 includes similar spectral energy distributions: blackbody fits to the USNO B, 2MASS, and WISE data give $3499 \mathrm{~K}$ for LHS 325 and 3184 for LHS 1691, which are similar to the fit for W0707+1705.

The sequence from ordinary red dwarf $(\mathrm{dM})$ through subdwarf (sdM), extreme subdwarf, and ultra-subdwarf is one of

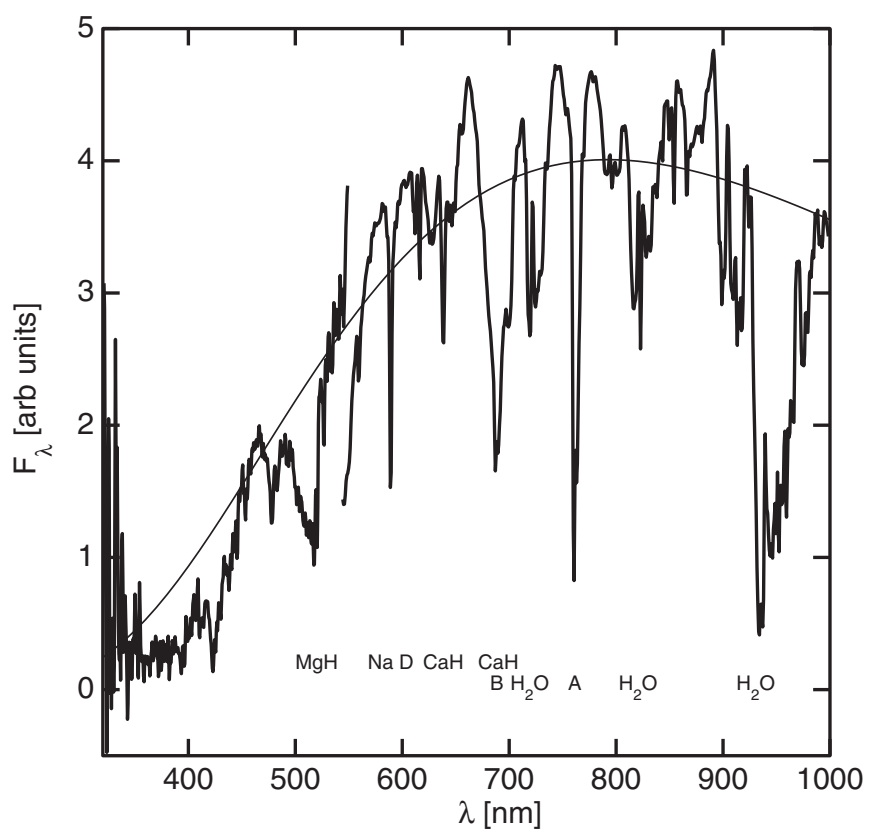

Figure 5. Palomar Double Spectrograph spectrum of W0707+1705, shown as the heavy dark line. The discontinuity near $550 \mathrm{~nm}$ is due to the dichroic separating the red and blue sides of the spectrograph. This spectrum has been smoothed to the resolution of the plot with a $1.5 \mathrm{~nm}$ FWHM smoothing kernel. The lower line of spectral identifications is due to telluric absorption bands. An arbitrarily scaled $3680 \mathrm{~K}$ blackbody is represented by the thin solid curve.

(Supplemental data (FITS) of this figure are available in the online journal.)



Figure 6. Full resolution blowup of the Na D line region of the W0707+1705 spectrum. The arrows indicate the rest wavelengths of the doublet.



Figure 7. Expanded view of the Ca II IR triplet part of the spectrum. 


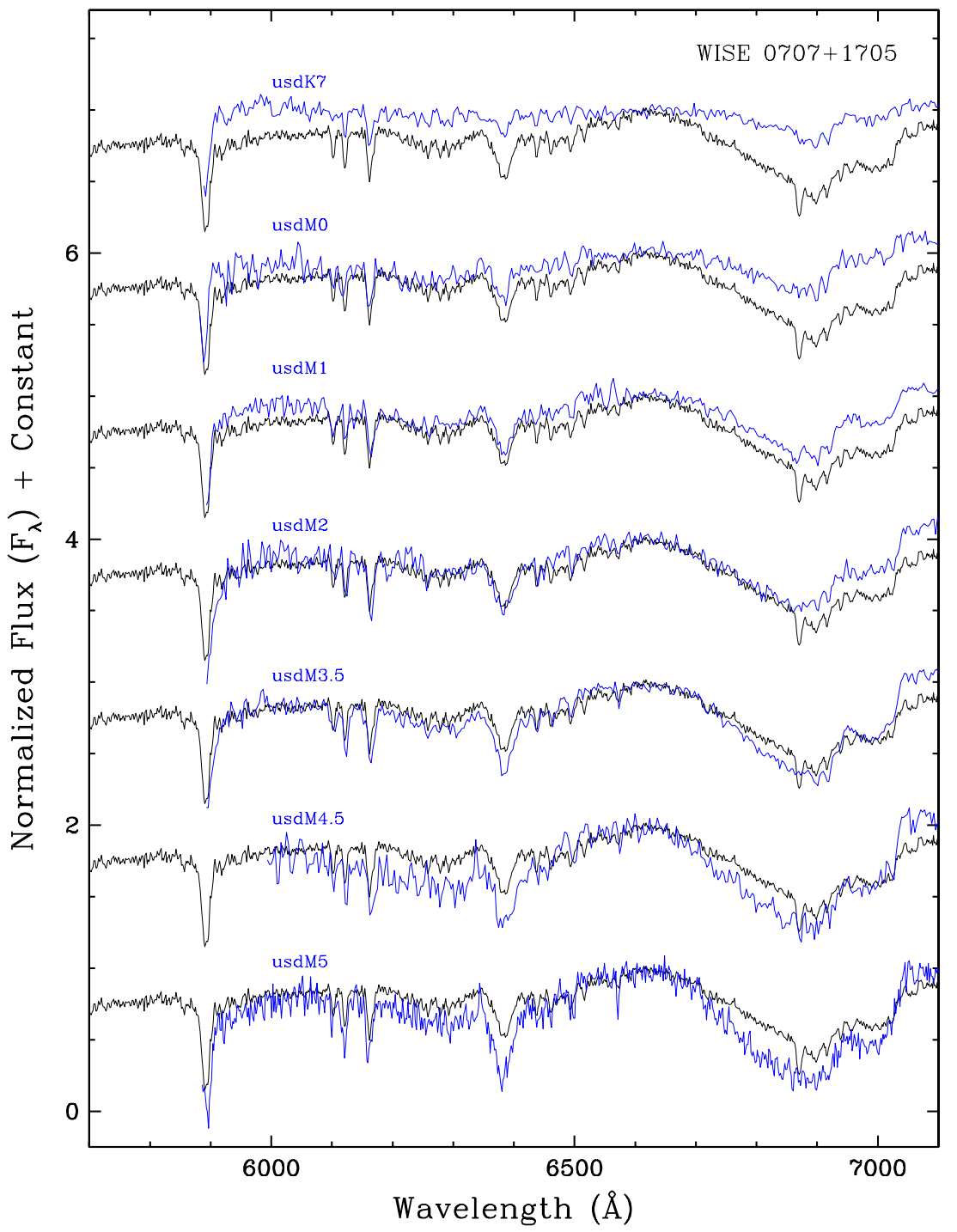

Figure 8. Spectrum of W0707+1705 compared to spectral standards from Lépine et al. (2007). All spectra are normalized at $660 \mathrm{~nm}$. (A color version of this figure is available in the online journal.)

weakening metal oxide bands relative to metal hydride bands and atomic lines. Thus, this is a sequence of decreasing metallicity, since to make a $\mathrm{TiO}$ molecule in a very low metallicity star requires two very rare atoms.

The near-infrared $\mathrm{CN}$ and $\mathrm{CO}$ bands are not seen in a spectrum obtained on UT 2013 September 15 with the TripleSpec instrument on the Apache Point Observatory $3.5 \mathrm{~m}$ telescope. The flux in the spectrum has been normalized using the 2MASS photometry. Figure 9 shows the spectrum divided by the blackbody fit in Figure 3. This is compared to a NASA IRTF SpeX prism spectrum of LHS 3768, which is tentatively classified as usdM3 based on the spectrum in Kirkpatrick et al. (1995). These spectra are very similar, and neither show any strong molecular bands. The $\mathrm{CN}$ band at $1.1 \mu \mathrm{m}$ in the spectrum of the carbon dwarf G77-61 shown by Joyce (1998) is at least ten times stronger than in W0707+1705. The iron abundance of G77-61 is very low, $10^{-4}$ of solar, but both carbon and nitrogen are enhanced relative to iron by a factor of 400 (Plez \& Cohen 2005). The weakness of any bands from carbon containing molecules in W0707+1705 suggests that carbon is depleted along with the metals in this source.

\section{DISCUSSION}

The spectral standards LHS 325 and LHS 1691 do not have published precise parallaxes so it is difficult to estimate the distance and absolute magnitude of W0707+1705. But Bochanski et al. (2013) give estimated absolute magnitudes for red dwarfs and subdwarfs seen by the SDSS (York et al. 2000). This paper defines a standard color $(g-r)_{\mathrm{dM}}$ for a red dwarf as a function of the $(r-z)$ color, and then uses the color excess $\delta(g-r)=(g-r)-(g-r)_{\mathrm{dM}}$ as an indicator of subdwarfism. Bochanski et al. (2013) then give fits for the absolutes magnitudes $M_{r, i, z}$ as a function of $(r-z)$ and $\delta(g-r)$. Unfortunately, we do not have SDSS griz photometry on W0707+1705, but we do have SDSS data on LHS 325 , with ugriz $=21.59,17.96,16.08,15.26$, and 14.81 in DR10 (Ahn et al. 2013). These data imply $\delta(g-r)=0.49$, appropriate for an ultra-subdwarf, and an absolute magnitude of $M_{r}=12.883 \pm 0.41$ based on the polynomial fits in Bochanski et al. (2013). If W0707+1705 has the same absolute magnitude, then the apparent $r$ magnitude (AB system) of $\approx 15.811 \pm 0.17$ from the CMC14 data implies a distance of $39 \pm 9$ pc and a 


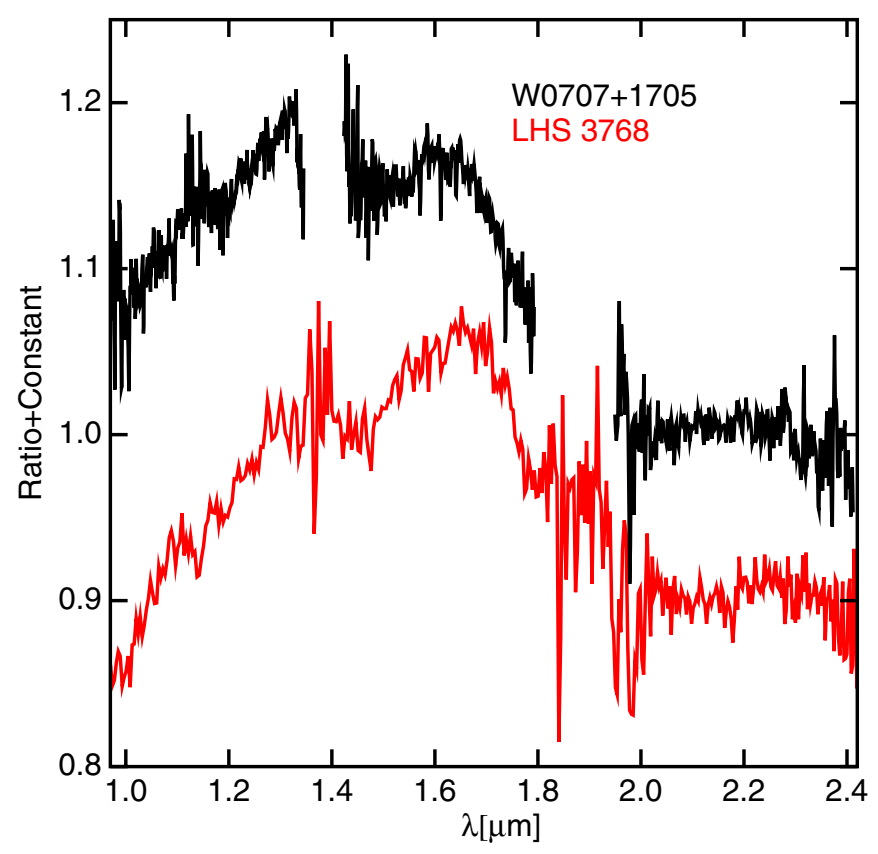

Figure 9. Triple-Spec spectrum divided by the blackbody fit, compared to a SpeX spectrum of LHS 3768. The spectra are normalized at $2.2 \mu \mathrm{m}$, and LHS 3768 is displaced downward by 0.1 for clarity.

(A color version of this figure is available in the online journal.)

tangential velocity of $v_{\tan }=330 \mathrm{~km} \mathrm{~s}^{-1}$. This seems unlikely, given the small radial velocity, but it is not ruled out. The parallax determination is consistent with $d=39 \mathrm{pc}$, but has such a large uncertainty that it does not improve our knowledge of the distance, except to impose a lower limit, $d>6 \mathrm{pc}$.

Note that the proper motion of W0707+1705 is primarily an

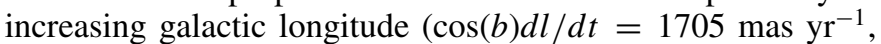
$d b / d t=555$ mas $\mathrm{yr}^{-1}$ ), as would be expected for a source not rotating with the Galaxy, since its location is near the anti-center $((l, b)=(199.29,11.13))$. The radial velocity of $-21 \mathrm{~km} \mathrm{~s}^{-1}$ with respect to the Sun gives $U V W$ components $(19,7,-4) \mathrm{km} \mathrm{s}^{-1}$, and the proper motion gives $(3.15 d,-7.46 d,+2.58 d) / \mathrm{km} \mathrm{s}^{-1}$ with $d$ in pc, while the Sun's velocity with respect to the local standard of rest (LSR) is $(9,12,7) \mathrm{km} \mathrm{s}^{-1}$ (Binney \& Tremaine 1987), so the total velocity with respect to the LSR is $(28+3.15 d, 19-7.46 d, 3+$ $2.58 d) \mathrm{km} \mathrm{s}^{-1}$.

If W0707+1705 is a member of a non-rotating singular isothermal sphere halo population, with $\sigma=v_{c} / \sqrt{2}$, where $v_{c} \approx 220 \mathrm{~km} \mathrm{~s}^{-1}$ is the local circular velocity, then the velocity distribution function is

$$
\begin{aligned}
f(U, V, W) & \propto \exp \left[-\left(U^{2}+W^{2}-\left(V+v_{c}\right)^{2}\right) / v_{c}^{2}\right] \\
& \propto \exp \left[-\left(1.2-0.070 d+0.00147 d^{2}\right)\right]
\end{aligned}
$$

The argument of the exponential is only -0.715 for $d=39 \mathrm{pc}$. If W0707+1705 is 1.1 mag less luminous than we have assumed, the argument of the exponential reaches its maximum value of -0.371 at $d=24 \mathrm{pc}$. This is equivalent to a Gaussian in $d$ with a mean of $24 \mathrm{pc}$ and a standard deviation of $18 \mathrm{pc}$.

But a singular isothermal sphere velocity distribution is an extreme assumption with an infinite escape velocity. Bochanski et al. (2013) also derive an ellipsoidal velocity distribution for usdM stars. For this distribution, the peak is at $d=15 \mathrm{pc}$ and $\sigma$ is $15 \mathrm{pc}$. This case is more in favor of W0707+1705 being two magnitudes more sub-luminous than an ultra-subdwarf star, but even the $d=39$ pc case with $v_{\tan }=330 \mathrm{~km} \mathrm{~s}^{-1}$ is only $1.7 \sigma$ out in the tail of this distribution.

For $d=39 \mathrm{pc}, \mathrm{W} 0707+1705$ is in a retrograde orbit around the Milky Way, and its speed with respect to the center of mass of the galaxy is no more than the local rotational speed. Thus, there is no indication that W0707+1705 is escaping from the Milky Way even if $v_{\tan } \approx 330 \mathrm{~km} \mathrm{~s}^{-1}$.

An accurate parallax would certainly be useful to distinguish between these scenarios for the luminosity and distance of W0707+1705. Improved optical photometry and spectroscopy would help to put W0707+1705 into the context of the many faint red stars seen by large surveys such as the SDSS.

\section{CONCLUSIONS}

W0707+1705 shows the power of the upcoming AllWISE data release to reveal odd denizens of the solar neighborhood. With an extremely short interval between epochs, the source association problems that have plagued previous searches for large proper motion objects are eliminated. Some examples based on discoveries within the last decade are: Teegarden's star (Teegarden et al. 2003), recovered with AllWISE proper motion values of $\mu_{\alpha}=3471 \pm 56 \& \mu_{\delta}=-3666 \pm 25$ mas yr $^{-1}$; WD 1339-340 (Lépine et al. 2005), recovered with $\mu_{\alpha}=-1916 \pm 78$ $\& \mu_{\delta}=999 \pm 84$ mas yr $^{-1}$; and Luhman's star (Luhman 2013), recovered with $\mu_{\alpha}=-2646 \pm 26 \& \mu_{\delta}=408 \pm 25$ mas yr $^{-1}$. These stars are all easily visible on optical sky survey plates but images at different epochs were not correctly associated. Previously known higher proper motion stars like Barnard's star and Kapteyn's star are also easily recovered. See Section II.6.d of the AllWISE Explanatory Supplement for more information on the AllWISE motion limits.

Based on sampling in test regions, we estimate that 2000-3000 real new proper motion detections will be found in the whole sky (Kirkpatrick et al. 2014). In addition to proper motions, AllWISE will provide a deeper survey of the sky at 3.4 and $4.6 \mu \mathrm{m}$, and new tools to study the variability of sources all over the sky.

We thank the referee, Ralf-Dieter Scholz, for a very fast and useful report pointing out the UCAC and CMC observations.

This publication makes use of data products from the Widefield Infrared Survey Explorer, which is a joint project of the University of California, Los Angeles, and the Jet Propulsion Laboratory/California Institute of Technology, funded by the National Aeronautics and Space Administration.

The WISE, 2MASS, and USNO B data were all provided by the Infrared Science Archive at Caltech.

The Digitized Sky Survey images were provided by the Space Telescope Science Institute.

Support for M.F. was provided by NASA through Hubble Fellowship grant HF-51305.01-A

This research has benefitted from the SpeX Prism Spectral Libraries maintained by Adam Burgasser at http://www.browndwarfs.org/spexprism.

Facilities: WISE, FMO:31in/FanCam, Hale (DoubleSpec), ARC (TSpec)

\section{REFERENCES}

Ahn, C. P., Alexandroff, R., Allende Prieto, C., et al. 2013, arXiv:1307.7735 Binney, J., \& Tremaine, S. 1987, Galactic Dynamics (Princeton, NJ: Princeton Univ. Press)

Bochanski, J. J., Savcheva, A., West, A. A., \& Hawley, S. L. 2013, AJ, 145, 40 Boyajian, T., von Braun, K., van Belle, G., et al. 2012, ApJ, 757, 112 
Evans, D. W., Irwin, M. J., \& Helmer, L. 2002, A\&A, 395, 347

Gizis, J. E., Troup, N. W., \& Burgasser, A. J. 2011, ApJL, 736, L34

Hamuy, M., Folatelli, G., Morrell, N. I., et al. 2006, PASP, 118, 2

Harris, H., Dahn, C., Vrba, F., et al. 1999, ApJ, 524, 1000

Joyce, R. R. 1998, AJ, 115, 2059

Joye, W. A., \& Mandel, E. 2003, in ASP Conf. Ser., 295, Astronomical Data Analysis Software and Systems XII, ed. H. E. Payne, R. I. Jedrzejewski, \& R. N. Hook (San Francisco, CA: ASP), 489

Kanneganti, S., Park, C., Skrutskie, M. F., et al. 2009, PASP, 121, 885

Kirkpatrick, J. D., Cushing, M. C., Gelino, C. R., et al. 2011, ApJS, 197, 19

Kirkpatrick, J. D., Henry, T. J., \& Simons, D. A. 1995, AJ, 109, 797

Kirkpatrick, J. D., Schneider, A., Fajardo-Acosta, S., et al. 2014, ApJ, in press

Lépine, S., Rich, R. M., \& Shara, M. M. 2005, ApJL, 633, L121

Lépine, S., Rich, R. M., \& Shara, M. M. 2007, ApJ, 669, 1235

Liu, M. C., Deacon, N. R., Magnier, E. A., et al. 2011, ApJL, 740, L32
Luhman, K. L. 2013, ApJL, 767, L1

Mace, G. N., Kirkpatrick, J. D., Cushing, M. C., et al. 2013, ApJS, 205, 6

Mainzer, A., Bauer, J., Grav, T., et al. 2011, ApJ, 731, 53

Martin, C. D., Bianchi, L., Donas, J., et al. 1999, in ASP Conf. Ser., 164 Ultraviolet-Optical Space Astronomy Beyond HST, ed. J. A. Morse, J. M. Shull, \& A. L. Kinney (San Francisco, CA: ASP), 182

Monet, D. G., Levine, S. E., Canzian, B., et al. 2003, AJ, 125, 984

Plez, B., \& Cohen, J. G. 2005, A\&A, 434, 1117

Skrutskie, M. F., Cutri, R. M., Stiening, R., et al. 2006, AJ, 131, 1163

Teegarden, B. J., Teegarden, B. J., Pravdo, S. H., et al. 2003, ApJL, 589, L51

Thompson, M. A., Kirkpatrick, J. D., Mace, G. N., et al. 2013, PASP, 125, 809

Wright, E. L., Eisenhardt, P. R. M., Mainzer, A. K., et al. 2010, AJ, 140, 1868

York, D. G., Adelman, J., Anderson, J. E., Jr., et al. 2000, AJ, 120, 1579

Zacharias, N., Finch, C., Girard, T., et al. 2013, AJ, 145, 44 